\title{
REINTERPRETANDO UN SITIO ARQUEOLÓGICO EN PAMPA SECA
}

\section{Guillermo Heider}

\author{
Laboratorio y Cátedra de Prehistoria y Arqueología. \\ Juan Filloy s/n, Ciudad Universitaria. 5000. Córdoba. guillermoheider@hotmail.com.ar \\ Presentado el: 22/04/2010 - Aceptado 24/07/2010
}

\section{Resumen}

En el año 2006 comenzaron a realizarse en el Departamento General Roca (pcia. de Córdoba), estudios arqueológicos sistemáticos. El abordaje inicial del área, carente de investigaciones previas, fue realizado con premisas propias de la Ecología del Comportamiento Humano o Ecología Evolutiva (amplitud de la dieta, selección de parcelas y teorema de valor marginal, etc.). De esta forma se intentó conseguir un panorama de base amplio sobre las relaciones entre los grupos cazadores-recolectores y su ambiente. La nueva información recolectada posibilitó la utilización de diferentes perspectivas o vías de entrada al registro para complementar los modelos que se están utilizando. Algunas de las hipótesis puestas en discusión desde La Etnoarqueologia resultaron de especial utilidad para el abordaje sobre los sitios, su materialidad y su contexto ideológico.

Palabras claves: Pampa Seca, Etnoarqueologia, Cazadores-recolectores, Reinterpretación

\begin{abstract}
Archaeological researches in General Roca Department (Province of Córdoba), began in 2006. The initial approach to the area, lacking previous investigations, was realized following The Ecology of the Human Behavior or Evolutionary Ecology premises (extent of the diet, selection of plots and theorem of marginal value, etc.). By this way a wide panorama about the relations between huntergatherers groups and the environment was obtained. The new gathered information make possible the utilization of different perspectives or routes of entry to the record to complement the models in use. Some of the hypotheses discussed from The Etnoarchaeology resulted of special usefulness for the sites the boarding, their materiality and their ideological context.
\end{abstract}

Key words: Pampa Seca, Etnoarchaeology, hunter-gatherers, Reinterpretation

\section{Introducción}

El estudio de los grupos cazadores-recolectores constituye un tema medular para los arqueólogos que hace más de cien años comenzaron a interesarse en la región pampeana. En este sentido, una de las ideas centrales que se mantuvo inalterable desde los comienzos de las investigaciones es aquella en la que se muestra a la región como gran área cultural, llegando profundamente arraigada hasta la década del 90. Sobre esta cuestión, Politis y Barros (2003-2004:62) señalan que "como unidad de análisis se basa en tradiciones de 
investigación y en cierto consenso contemporáneo más que en características ecológicas o arqueológicas distintivas".

La cita antes mencionada es de gran influencia en el proyecto de investigación que se está realizando. Aun cuando se enfrenten las problemáticas del registro desde una perspectiva macro que podría ser de "arqueólogos de la región pampeana", por otra parte, se intenta exponer las características que le son propias a la región Norte de Pampa Seca, subregión arqueológica del Caldenar (sensu Berón y Politis 1997).

A partir del año 2006 comenzaron a realizarse en el Departamento General Roca, Córdoba, una serie de estudios sistemáticos. Una de las proposiciones básicas del trabajo que allí se desarrolla consiste en contribuir a llenar el vacío de información que existe en el norte de la región pampeana y analizar los posibles marcadores de articulación con los grupos de las regiones vecinas (Sierras Centrales, Cuyo, Centro de La Pampa y Oeste de Bs. As y Santa Fe).

Se entiende que un abordaje inicial conciso a un área carente de investigaciones puede ser logrado con algunas de las premisas más utilizadas por La Ecología del Comportamiento Humano o Ecología Evolutiva (amplitud de la dieta, selección de parcelas y teorema de valor marginal, etc.). De esta forma se intenta conseguir un panorama de base amplio sobre las relaciones entre los grupos cazadores-recolectores y su ambiente. Los trabajos de Winterhalder y Smith (1981); Bettinger (1991); Boone y Smith (1998), entre otros, se complementan con estudios sobre problemáticas regionales y extra regionales de alta movilidad, abastecimiento de materias primas, interacción y complejidad social entre otros.

Durante cuatro temporadas de campañas sistemáticas se prospectaron las márgenes de 17 lagunas y se realizaron sondeos en 4 de los 25 sitios superficiales detectados. Con este nuevo panorama, en el que se presenta un cuerpo mayor de datos, es posible agregar otras perspectivas o vías de entrada al registro que complementen y/o reenfoquen la mirada inicial que se planteo al comienzo de las investigaciones. Algunas de las teorías o modelos puestos en discusión desde la etnoarqueologia resultan de especial utilidad para este nuevo abordaje sobre los sitios, su materialidad y su contexto ideológico.

En la naciente del arroyo Boro Hué se encuentra el sitio arqueológico La Olla. Está ubicado a pocos metros de una vertiente de agua dulce del mismo nombre. Conocido desde los años 70, fue objeto de una intensa recolección superficial por un aficionado durante muchas temporadas. Luego fue incluida en el marco del proyecto arqueológico para realizar prospecciones. En los meses de marzo y abril de 2010 se realizaron sondeos estratigráficos. Los resultados del análisis de los materiales de colección, de los obtenidos en prospecciones y en niveles estratigráficos sirven para cuantificar y discutir el presente trabajo.

\section{Ambiente en el arroyo}

El arroyo Boro Hué se encuentra ubicado en el SurOeste del Departamento General Roca. Es una cuenca endorreica de curso meandroso y ciclo principalmente estival que suele perderse en parte de su recorrido a su desembocadura (principalmente durante los meses de otoño e invierno). El desagüe de la cuenca se da en la laguna Rali-có, donde se encuentran 2 de los 25 sitios superficiales detectados hasta el momento. 


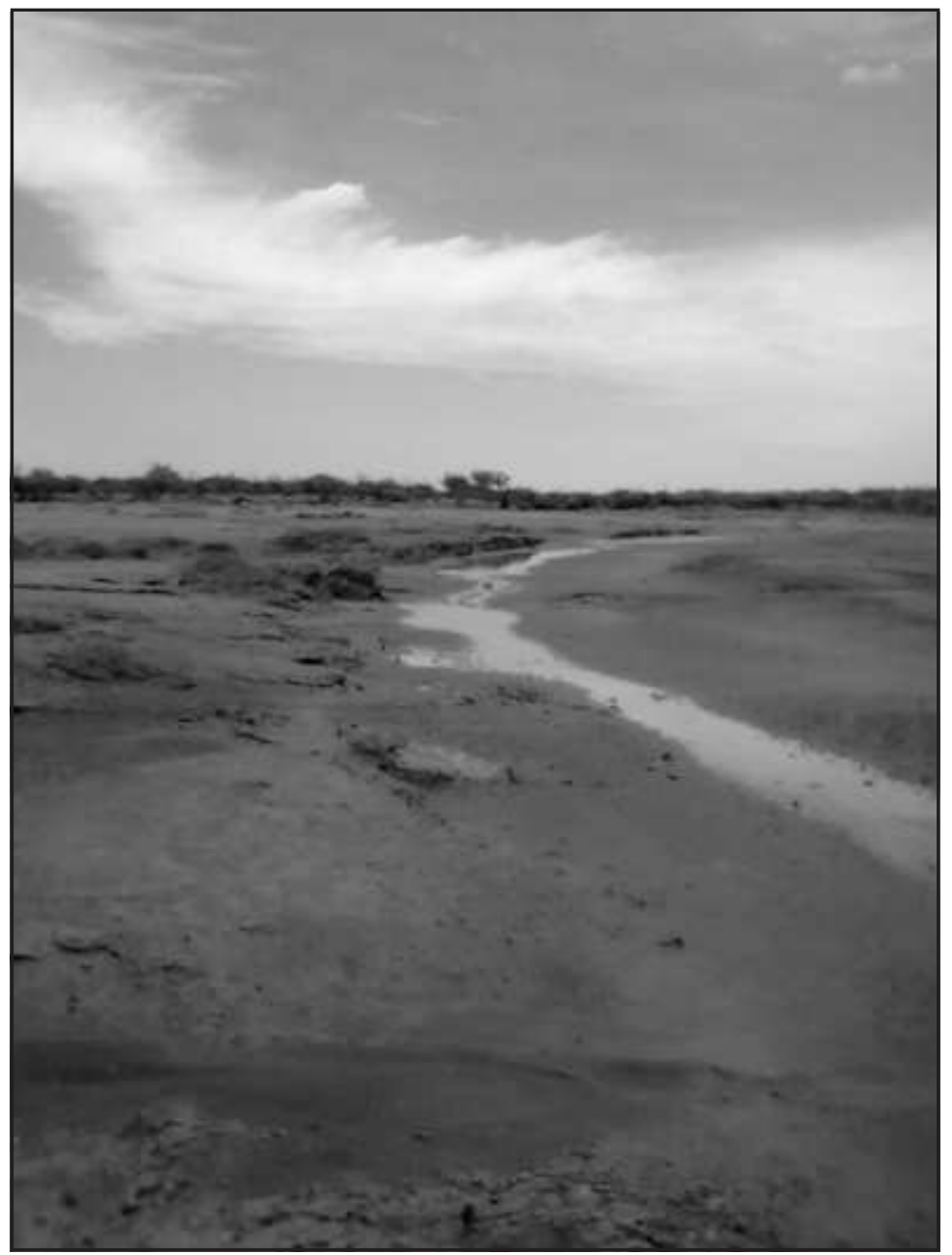

Figura 1. Vista del arroyo Boro Hué, desde el Sur hacia la vertiente donde se forma el arroyo, a ambas márgenes del arroyo se encuentran restos arqueológicos en posición superficial.

El sitio, el arroyo y las lagunas se encuentran ubicados en lo que geomorfológicamente se conoce como Pampa Medanosa, constituida por un relieve ondulado generado por una sobreimposición de formas medanosas parcialmente estabilizadas bajo las condiciones climáticas actúales. La erosión eólica es el proceso dominante en el área fuertemente asociadas a procesos desborde y sedimentación producto de ciclos hídricos de los arroyos. El clima presenta un amplio rango de oscilación térmica, con frecuentes heladas invernales y deficiencia hídrica de este a oeste por evapotranspiración durante el verano. Las isohietas son de 350 a $580 \mathrm{~mm}$ con predominancia de las lluvias estivales.

La región se corresponde con el Distrito del Caldén, dentro de la Provincia Fitogeográfica del Espinal también llamada de Monte Alto (Cabrera 1976). Acompañan al caldén (prosopis caldenia) árboles como el algarrobo (prosopis flexuosa var. flexuosa) y el chañar (Geoffroea 
decorticans), además de numerosos arbustos como el piquillín (Condalia microphylla), y el alpataco (prosopis flexuosa var. depresa). Especies autóctonas como el guanaco (Lama guanicoe), y el venado de las pampas (Ozotoceros bezoarticus) han desaparecido, sin embargo subsisten en el monte relictual de este sector del caldenar la vizcacha (Lagostomus maximus), el peludo (Chaetophractus vellorosus), el ñandú (Rhea americana) y el zorro (Lycalopex culpaeus).

\section{El sitio}

Ubicado a ambas márgenes del arroyo Boro Hué se encuentra el sitio de recolección

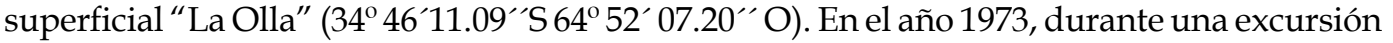
de caza, el sitio fue descubierto por un aficionado quien realizo campañas de recolección en reiteradas oportunidades. Como resultado de las visitas existe una colección que cuenta con 36 instrumentos, 25 lascas, 172 desechos de talla y esquirlas, una conana, dos manos de moler, tres instrumentos de hueso y un conjunto muy deteriorado de restos óseo humanos (fragmentos del cráneo, dos huesos largos fracturados y un par de metatarsos).

En el año 2006 comenzaron las prospecciones arqueológicas sistemáticas, seguida por nuevas campañas en 2007 y 2008. El diseño de prospección incluye aspectos metodológicos tomados de los autores Orton 2000; Borrero y Nami 1996; Dunnell 1992, adaptados a las particularidades del emplazamiento. La definición de Borrero et al. (1992) quienes toman una concentración de artefactos mayor a $0.08 \mathrm{~m} 2$ como el mínimo para definir un sitio arqueológico fue el punto de base para definir los límites del sitio durante las prospecciones.

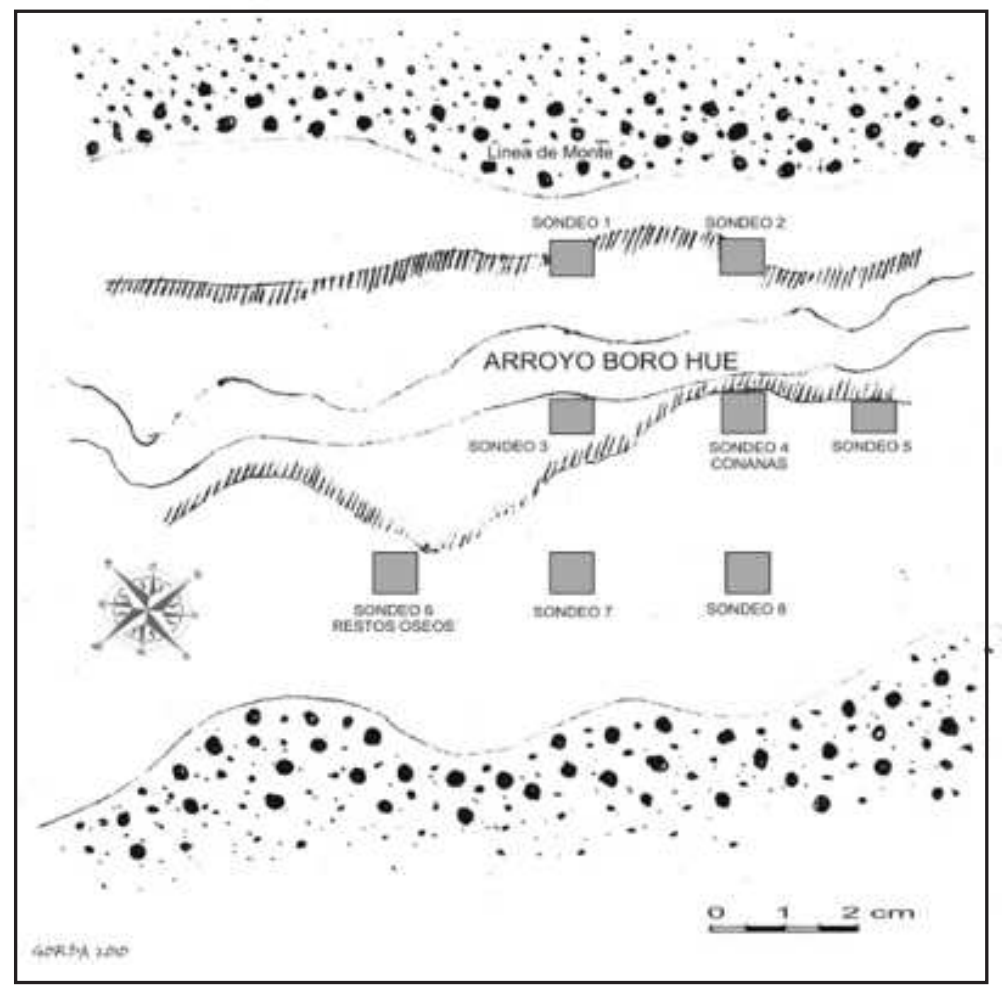

Figura 2. Plano de los sondeos realizados en La Olla 
Sin embargo es clara la dificulta de definir limites concisos en base a la distribución espacial de los elementos, como señalan Ebert et al. (1987).

\begin{tabular}{|c|c|c|c|c|}
\hline $\begin{array}{l}\text { Numero } \\
\text { de pieza }\end{array}$ & Grupo Tipológico & Materia Prima & $\begin{array}{c}\text { Situación de } \\
\text { Lascado }\end{array}$ & Serie técnica \\
\hline LO 1 & Raspador & Calcedonia & Unifacial directa & Retoque marginal \\
\hline LO 2 & Raedera & Calcedonia & Unifacial directa & Retoque ultramarginal \\
\hline LO 3 & Punta de flecha & Cuarzo & Bifacial & Ret. ext., y marginal \\
\hline LO 4 & Bola boleadora & Tos. Calcárea & 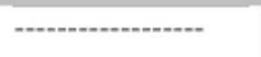 & Picado pulido abrasión \\
\hline LO 5 & Raspador & Cuarcita & Unifacial inversa & Retalla y ret. Marginal \\
\hline LO 6 & Raedera & Calcedonia & Unifacil directa & Microretoque marginal \\
\hline LO 7 & Punta de flecha & Calcedonia & Bifacial & Retoque ext., y marginal \\
\hline LO 8 & Bola boleadora & Arenisca & - & Picado pulido abrasión \\
\hline LO 9 & Filo retocado & Cuarcita & Unifacial directa & Retoque ultramarginal \\
\hline LO 10 & Raspador & Calcedonia & Unifacil directa & Retoque marginal \\
\hline LO 11 & Punta de flecha & Cuarzo & Bifacial & Ret. ext., y marginal \\
\hline LO 12 & Conana & En estudio & - & Picado pulido abrasión \\
\hline LO 13 & Conana & En estudio & -..- & Picado pulido abrasión \\
\hline LO 14 & Conana & En estudio & - & Picado pulido abrasión \\
\hline LO 15 & Bola Boleadora & Arenisca & 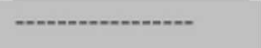 & Picado pulido abrasión \\
\hline
\end{tabular}

Tabla 1. Instrumentos recuperados en La Olla, arrollo Boro Hué

Para el diseño de las transectas se tuvo en cuenta la profundidad del arroyo $(10 \mathrm{~cm}$ en la parte más profunda) y su ancho de costa a costa (1.30 m como máximo). Las líneas fueron trazadas en dirección Este-Oeste para que corten perpendicularmente al arroyo, el ancho de las mismas fue de $1 \mathrm{~m}$. y fueron espaciadas entre si cada $3 \mathrm{~m}$. El largo de las transectas no fue homogéneo, ya que en el diseño de prospección se incluyeron las barrancas adyacentes al arroyo. Esto permite abarcar un mayor área de trabajo y del mismo modo se cuantificaron los procesos erosivos formadores de paisaje que modifican los límites del sitio y la distribución espacial de los artefactos.

El proceso de salinización de la superficie del arroyo producido por la ausencia de lluvias en el año 2009 impidió la realización de prospecciones. En esa temporada se plantearon y realizaron sondeos estratigráficos con el fin de evaluar el potencial arqueológico del sitio. Se realizaron 8 sondeos estratigráficos de de $50 \mathrm{~cm}$. x $50 \mathrm{~cm}$. con una profundidad variable siguiendo los limites de los horizontes de suelo hasta la tosca calcárea o el nivel de la freática. 


\begin{tabular}{l|l|l|l|l|l|l}
\hline Tamaño & \multicolumn{4}{c}{ Estado de Fragmentación } & \multicolumn{2}{c}{ Total } \\
\hline & LENT & LFCT & LFST & DNC & INDI & \\
\hline Muy pequeño & 2 & 15 & 7 & 12 & 5 & 41 \\
\hline $\begin{array}{l}\text { Pequeño } \\
\text { Med. Pequeño }\end{array}$ & 9 & 21 & 36 & 41 & 37 & 144 \\
\hline Med. Grande & 1 & - & 1 & 26 & 35 & 63 \\
\hline Grande & - & - & - & - & - & - \\
\hline TOTALES & - & - & - & - & - & - \\
\hline
\end{tabular}

Referencias: LENT=lascas enteras, LFCT=lascas fracturadas con talón, LFST=lascas fracturadas sin talón, $\mathrm{DNC}=$ desechos no clasificables, $\mid \mathrm{NDI}=$ indiferenciadas.

Tabla 2. Desechos de talla y estado de fragmentación

\section{Resultados de las prospecciones y sondeos}

Con el objetivo de poder realizar un manejo más sencillo y claro de los resultados se tuvo en cuenta a todas las campañas de prospecciones como un cuerpo único de datos. En las tres campañas de prospecciones se realizaron un número estadísticamente similar de hallazgos, constituido mayormente por desechos de talla, lascas y algunos instrumentos confeccionados en materias primas aloctonas (con excepción de una bola de boleadora realizada en la tosca calcárea del lugar). Durante las campañas se pudo observan la presencia de un escaso número de restos óseos semi-enterrados los cuales fueron tenidos en cuenta al momento de diseñar los sondeos estratigráficos.

Los sondeos tuvieron un diseño que fue modificado durante la campaña, luego de constatarse que el arroyo había comenzado a descubrir una conana. De los ocho sondeos realizados cinco tuvieron resultados negativos. En uno de los sondeos con hallazgos se rescataron 2 raspadores, una punta de flecha y un total de 45 restos entre desechos de talla y lascas; en otro se encontró una acumulación de huesos cuyo límites lateral e inferior no está claro aun y en el tercero se encontraron los mencionados instrumentos de molienda asociados a otros dos similares. Los siguientes cuadros resumen los restos arqueológicos recuperados y su posterior estudio en laboratorio, para el cual se utilizaron algunas de las categorías propuestas por Aschero $(1975,1983)$. Por otra parte las conanas se encuentran aun en el Laboratorio de la Cátedra de Prehistoria y Arqueología de la UNC donde se les realizaran análisis de fitolitos y petrográficos.

A diferencia de la colección, en que solo se encontraron 3 restos óseos no humanos (una aguja, dos perforadores, todos confeccionados en huesos de ave sin identificación), en las campañas de prospecciones realizadas desde 2006 se constataron hallazgos de este tipo. El sesgo de recolección por parte del aficionado es muy claro en este punto, la ausencia de de los elementos óseos en el registro son consecuencia de la confusión de los huesos de guanaco con los de ovejas (ovis capri) de los peones del establecimiento rural donde se encuentra el sitio (Aficionado, com. pers.). 


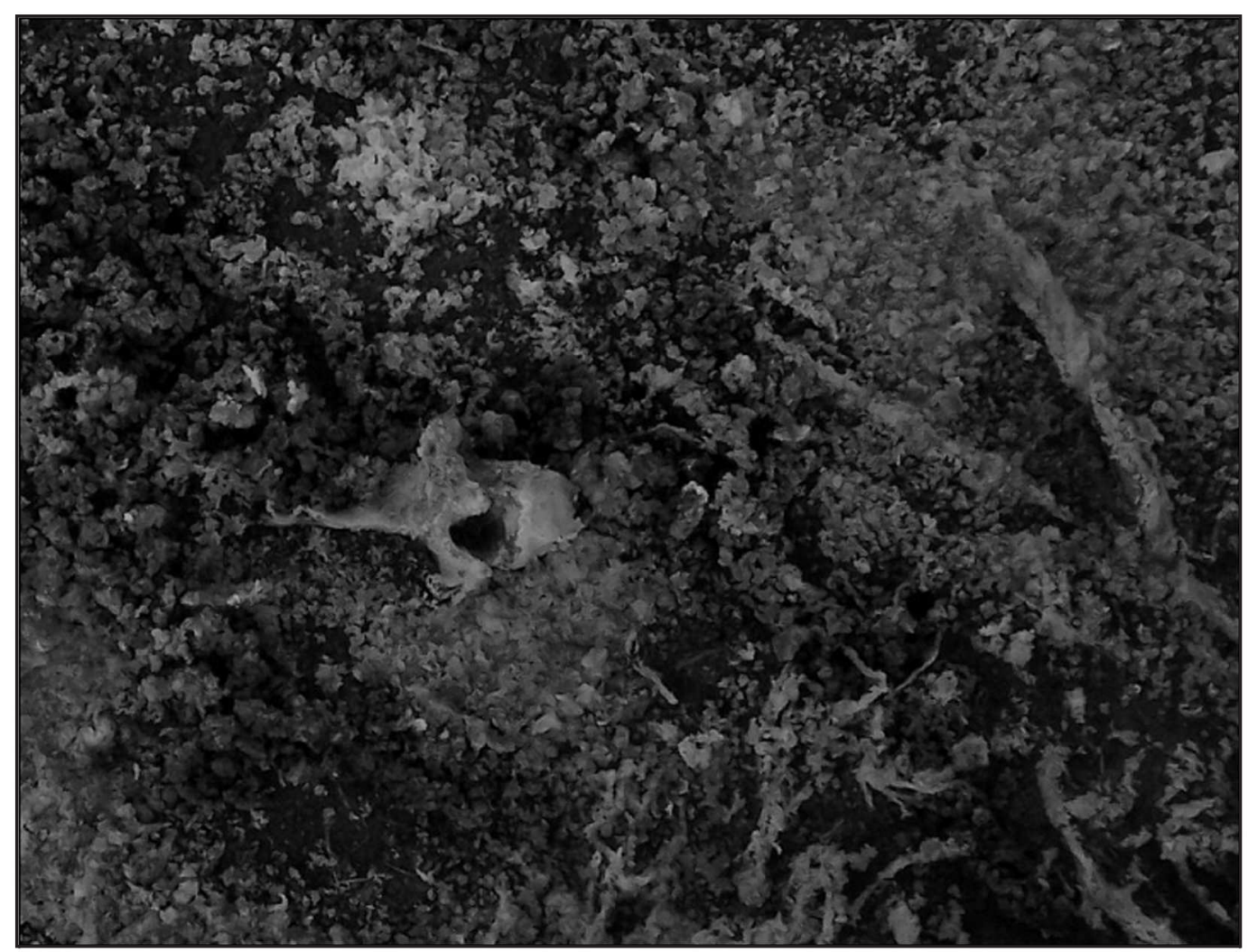

Figura 2. Restos Óseos en posición superficial, recuperados en durante las prospecciones

\section{Análisis arqueofaunístico}

El análisis arqueofaunistico mostro que la totalidad de los restos obtenidos en recolección superficial, sumados a los recolectados durante el sondeo del sitio, corresponden a guanaco (lama sp.). Los restos aun están siendo estudiados pero en primera instancia puede exponerse que se recuperaron 6 fragmentos mandibulares, 22 vertebras, 3 escapulas, 5 humeros, 12 fémures y 7 tibias. Por otra parte suelen encontrarse durante las prospecciones, y no es " $\mathrm{La}$ Olla" una excepción, gran cantidad de huevos de ñandú fragmentados (Rhea americana) asociados a los restos líticos en diferentes sectores de los sitios superficiales. Particularmente en el lugar de hallazgos de guanaco no se encontró otro tipo de restos óseos, ni siquiera cascara de huevo.

\section{Una nueva mirada al registro arqueológico}

En un trabajo anterior (Heider 2009) se expusieron resultados de micro-región "el Cuero", donde se encuentra el sitio "La Olla". En aquel trabajo se exhibieron una serie de conclusiones orientadas desde los postulados utilizados por la Ecología Evolutiva. Se planteo el uso de estrategias tecnológicas conservadas, con maximización en el uso de instrumentos en directa relación con la ausencia de materias primas aptas para la talla. En este sentido se interpreto al sitio como un campamento de base residencial donde tenían lugar todas las etapas de la manufactura de instrumentos, desde la reducción de los núcleos al reactivado de filos. Por 
otra parte la presencia de conanas y manos parecerían indicar, en principio, un aprovechamiento del monte de caldén circundante.

El contar con un cuerpo mayor de datos recolectados sistemáticamente permite precisar límites más claros tanto del sitio como de los restos arqueológicos dentro del mismo. Esta nueva información permite nuevos análisis que re-orientan nuestra estrategia de investigación, un nuevo punto de vista sobre "La Olla" puede ser expuesto desde los postulados básicos de la etnoarqueologia. La búsqueda de relaciones recurrentes entre la conducta humana y la cultura material constituye uno de los tres campos donde la etnoarqueologia es útil para interpretación arqueológica (sensu Politis 2004). Algunos aportes en este sentido pueden ser esbozados a nivel de hipótesis inicial dentro del presente trabajo.

A partir de esta mirada "etnoarqueologica", algunos de los puntos que previamente observamos como partes de una serie técnica de manufactura de artefactos o un locus de desecho de restos óseos toman una dimensión distinta. Cuestiones ideológicas y patrones de pensamiento propios de ese grupo pueden comenzar a explorarse con mayor profundidad en este y otros sitios. Se prestó especial atención para este trabajo a dos puntos específicos del registro, los cuales pueden considerarse reinterpretados, o por lo menos observados desde una nueva vía de análisis.

En primer lugar nos referimos a la acumulación de hueso de guanaco, (ignorada por el aficionado), prospectada y sondeada en diferentes campañas por parte del equipo arqueológico. En una primera mirada podría esgrimirse como procesos formadores de esta acumulación algunos factores tafonomicos no humanos como la acción del agua, carnívoros y/o roedores. Largamente tratada en la bibliografía arqueológica (Hanson 1980; Behrensmeyer 1975; Binford 1981, etc.), la acción del agua no parece un factor decisivo en esta acumulación debida a la baja dinámica hidráulica del arroyo que presenta un marcado régimen estival con bajo caudal aun en los momentos más lluviosos del año. Por otra parte la presencia de carnívoros o roedores actuando como factores de transformación de los sitos no parecen tener injerencia decisiva en este caso, la ausencia de las marcas dejadas por estos en los huesos y a la falta de cuevas en el este sector del sitio apoyarían empíricamente esta idea.

La problemática sobre acumulaciones diferencial de restos óseos fue tratada por diferentes autores en sociedades cazadoras. Los trabajos de Binford $(1978,1981)$ muestran la presencia de áreas especializadas de desecho de restos de ungulados en los campamentos Nunamiut. Por su parte Politis (2002) observo entre los Hotï diversos locus de acumulación de desecho en los campamentos residenciales. Específicamente en la región pampeana existen trabajos que hacen referencia a esta cuestión, por ejemplo en el sitio Paso Otero 3 (Martínez 1999). Los argumentos expuestos en estos y otros trabajos, sumandos a la ausencia de factores tafonomicos actuando claramente en la acumulación ósea en el sitio La Olla, indicarían una fuerte presencia de la acción antropica en el proceso de acumulación discreta de los restos óseos de guanaco.

El otro punto sobre el que se intenta una nueva vía de análisis es la presencia en el sitio de dos de las tres bolas de boleadoras halladas, que en primera instancia marcamos como mal elaboradas o en proceso de elaboración. Como sucede con el resto de los instrumentos líticos hallados en toda la sub-región, la ausencia de canteras con materias primas adecuadas implicaría técnicas de manufactura donde se ponga especial cuidados en la manufactura, minimizando los riegos de fractura, de defectos en la confección y una reutilizando las mismas en caso de fractura. 
La mayor parte de las bolas de boleadora recuperadas por aficionados y por los investigadores en esta subregión (33 de 38 artefactos recuperados) fueron halladas fuera de los sitios arqueológicos, probablemente como resultado de la pérdida durante las excursiones de caza. Son claras las diferencias en la confección de estas dos bolas irregulares y el resto de los instrumentos encontradas. La diferencia no es solo en la terminación dada por la técnica de elaboración sino que también es distinto el material sobre el que se confecciono una de las bolas.

En ningún caso conocido para la micro-región se utilizo la roca calcárea propia de los afloramientos del Sur de Córdoba para la manufactura de instrumento, debido a la excesiva hidratación y las malas condiciones para la talla, sin embargo como ya se menciono, una de las dos bolas fue realizada en este material. Esta forma de realizar las bolas y el material no se corresponden con las expectativas que plantean los modelos de la Arqueología Evolutiva. En principio, para la bola de arenisca, es esperable un marcado esfuerzo en la elaboración y conservación en estos instrumentos de larga vida útil potencial con abastecimiento costoso a nivel energético, y por otra parte la bola de tosca calcárea no cuenta con la dureza aparente para realizar las funciones de caza con las que se creía fue elaborada.

Una explicación que no incluya solamente postulados netamente tecno-morfologicos cuando se describen estos instrumentos puede ser introducida inicialmente en este trabajo. Existen toda una serie de trabajos etnográficos donde se describe el uso de las bolas en ceremonias, juegos, etc., pudiendo encontrarse estas bolas utilizadas en acciones más ligadas a la esfera ideacional que a la esfera utilitaria de la caza (para las que parecen poco aptas). Un claro ejemplo es la utilización de las boleadoras en el sutee de Paine, realizado en Leuvuco por orden de su hijo Calvaiu, donde fueron utilizadas para sacrificar no solo animales sino también mujeres lonco y machis acusadas de su muerte. Otra posible vía de análisis podría incluir a estos instrumentos dentro de la producción material de los niños, siendo estos los que realizaron la confección de las bolas como parte del proceso de aprendizaje o juegos de imitación de sus mayores.

\section{Discusión}

La arqueología del Norte de la Región de Pampa Seca se encuentra aún en un estado inicial de su desarrollo, sin embargo luego de realizadas sucesivas campañas el material recuperado permite comenzar a realizar distintos tipos de abordaje al registro arqueológico, tomando diferentes perspectivas teóricas que complementen los resultados.

Los primeros trabajos sobre el área fueron fuertemente influenciados por los postulados de la arqueología evolutiva, intentando explicar el registro arqueológico usando modelos que relación de los cazadores-recolectores con el ambiente en términos de consumo y retorno energético. Sin dejar de lado estos modelos, que siguen siendo de utilidad para describir la relación hombre-ambiente, se comenzó a explorar vías alternativas de análisis de los sitios.

La realización de una mirada desde la Etnoarqueologia sobre La Olla no modifica algunas de las conclusiones iniciales realizadas con una mirada evolutiva. La idea sobre la presencia de un campamento de base residencial en la que se están llevando a cabo todas las tareas de la serie de técnica de confección de instrumento, como así también tareas de trozado y cocción de alimentos siguen vigentes. La posible presencia de niños confeccionando artefactos 
reforzaría este postulado, teniendo en cuenta que es de esperar en estos grupos una mayor movilidad logística en excursiones de caza por parte de adultos, quedando entonces los niños en los campamentos base donde realizarían los artefactos en forma de juego y como parte del aprendizaje.

Los referentes etnográficos que muestran a las bolas de boleadoras involucradas en actividades ceremoniales, diferentes de la caza, sumados a los estudios sobre la participación de los niños en la creación de restos materiales en los campamentos base son una buena vía de análisis para encarar una explicación algo diferente, y complementaria, del sitio La Olla. Por otra parte, la presencia de una acumulación ósea con una sola taxa no es nueva en el registro arqueológico pampeano. Para los sitios de la región en general, y para este sitio en particular se puede estar ante la presencia de un tratamiento diferencial de la basura en estos grupos de cazadores, seguramente con una fuerte presencia de cuestiones ideológicas subyacente.

No pueden plantearse, más allá del nivel inicial de hipótesis, ni la ritualidad en la elaboración y uso de las bolas de boleadora ni la presencia de niños en la elaboración de las mismas (mas aun teniendo en cuenta la ausencia de otros ítems de la tecnología con la misma problemática de elaboración). Como tampoco puede tener carácter definitivo la presencia de un trasfondo ideológico en el descarte de guanaco, pero el hecho de comenzar a bosquejar este tipo de cuestiones para el registro abre la puerta a una mirada más amplia y complementaria del sitio.

\section{Agradecimientos}

Deseo agradecer a mi director, Dr. Eduardo Berberián y al Dr. Diego Rivero por las sugerencias realizadas. A mis compañeros del Laboratorio de Prehistoria y Arqueología de la Universidad Nacional de Córdoba y a Juan, Efraín y Marcos por su colaboración constante.

\section{Bibliografía citada}

Aschero, C.

1975. Ensayo para una clasificación morfológica de artefactos líticos aplicada a estudios tipológicos comparativos. Informe al CONICET.

1983. Ensayo para una clasificación morfológica de artefactos líticos. Apendice A y B. Informe al CONICET.

Berberián, E.; F. Roldán

2001. Arqueología de Sierras Centrales. Historia Argentina Prehispánica. Tomo II: 937-948. Ed Brujas. Córdoba.

Berón, M. y G. Politis. 1997.

Arqueología Pampeana en la década de los'90. Estado de las investigaciones y perspectivas. En Arqueología de la Región Pampeana en la Década de los '90.M. Berón y G. Politis (eds.), pp. 732. Museo de San Rafael-INCUAPA, San Rafael, Mendoza.

Behrensmeyer, A.

1978. Taphonomic and ecolgic information from bone weathering. Paleobiology. 4: 150-162. 
Bettinger, R.

1991. Hunter-gatherers: archaeology and evolutionary theory. Plenum Press, New York.

Binford, L.

1978. Nunamiut Ethnoarchaeology. Academic Press, New York.

1981. Bones: ancient men and modern myths. Academic Press, New York.

Borrero, L.

1988. Estudios tafonómicos en Tierra del Fuego: su relevancia para entender procesos de formación del registro arqueológico. En Arqueología Contemporánea Argentina. Actualidad y perspectivas: 13-32. Ediciones Búsqueda.

Borrero, L.A. y H.G. Nami

1996. II) Piedra del Águila: Análisis de los materiales de superficie. Prehistoria, 2: 19-34.

Borrero, L.A., J.L. Lanata y B.N. Ventura

1992. Distribución de hallazgos aislados en Piedra del Águila. En Análisis espacial en la Arqueología Patagónica, L. A. Borrero y J. L. Lanata (eds.), pp. 9-20. Ediciones Ayllu, Buenos Aires.

Boone, J. y E. Smith.

1999. Is it evolution yet? A critique of evolutionary archaeology. Current Anthropology 39:141173.

Cabrera, A. L.

1976. Fitogeografía de la República Argentina. En Bol. Soc. Argentina de Botanica, 14 (1-2): 142. 7 lam.

Curtoni, R.; M. Lezcano y M. Fernández.

1996-1998. Prospección y rescate en el norte de La Pampa. El sitio arqueológico La Magdalena. Palimpsesto 5: 138-150.

Dunnell, R.

1992. The Notion Site. En Space, Time and Archaeological Landscapes, J. Rossignol y L. A. Wandsnider (eds.), pp. 21-41. Plenum Press, New York.

Hanson, C.B.

1980. Fluvial Taphonomic Processes: Models and Experiments. En Fossils in the Making, A. K. Behrensmeyer y A. P. Hill (eds.), pp.156-181. The University of Chicago Press, Chicago.

Harris, E. C.

1979. Principios de estratigrafía arqueológica. Ed. Crítica. Madrid.

Heider, G.

2009. Investigaciones arqueológicas en el área noroccidental de la región pampa seca, primeras aproximaciones al registro de la microrregión el Cuero "(Dpto. Gral. Roca, Córdoba)" En: Revista arqueológica de zonas aridas, UNRC. Tomo 1. 
Köppen, W.

1931. Climatología. En Fondo de Cultura Económica. Bs As.

Martínez, G.

1999. Tecnología, subsistencia y asentamiento en el curso medio del Río Quequén Grande: un enfoque arqueológico. Tesis Doctoral inédita, Facultad de Ciencias Naturales y Museo UNLP, La Plata.

Martínez, G y M. Gutiérrez.

2004. Tendencias en la explotación humana de la fauna durante el Pleistoceno finalHoloceno en la Región Pampeana (Argentina). ArchaeoZoologia XII. Zooarchaeology of South America. G. Mengoñi Goñales (ed.) bar international Series 1298. 81-98.

Orton, C.

2000. Sampling in Archaeology. Cambridge Manuals in Archaeology. Cambridge.

Politis, G.

1998. Arqueología de la infancia: una perspectiva etnoarqueológica. Trabajos de Prehistoria, 55 (2): 5-19.

2002. Etnoarqueología entre los Hotï (Cuenca del Orinoco, Venezuela). Implicancias para la arqueología pampeana. Conferencia dictada en el 3er Congreso de Arqueología de la Región Pampeana Argentina. Olavarría.

Politi, G. y P. Barros

2003-2004. La región Pampeana como unidad espacial de análisis en la arqueología contemporánea.En Folia Histórica del Nordeste $\mathrm{N}^{\circ} 16$, Resistencia.

Renfrew, C. y P. Bahn

1998. Archaeology. Theories, methods and practice. Thames and Hudson, Londres.

Schiffer, M.

1987. Formation Processes of the Archaeological Record. Univ. Of New Mexico Press, USA.

Winterhalder, B. y E. Smith.

1992. Evolutionary ecology and the social sciences. En: Ecology and human behaviour. Smith, E. y B. Winterhalder (Eds.) Evoltionary Pp. 3-23. Aldine De Gruyter. New York. 